\title{
Les échelles de gravité sur les inondations : réflexion nationale et exemple dans l'Hérault
}

\author{
The French national scale of flood magnitude and an example \\ on the Herault catchment
}

\author{
Michel Lang ${ }^{1}$ \\ Cemagref, Lyon
}

Roland Claudet ${ }^{2}$

Diren Languedoc-Roussillon, Montpellier

This paper presents two scales of flood magnitude. The former is related to a national French project for a systematic policy of experience feedback after natural hazard events. Combination of two rankings, through a national magnitude scale and a national damage scale will provide a better knowledge of the evolution of natural risk. The latter is related to flood mitigation actions on the upper part of the Herault catchment. Emergency action plans have been prepared, taking into account both the vulnerability and the magnitude class of a forecasted event.

\section{I 口 INTRODUCTION}

La succession à intervalles rapprochés ces dernières années de catastrophes liées aux inondations a conduit à s'interroger sur les raisons de cette aggravation et les remèdes possibles. Lorsque l'on cherche à analyser en retour les événements passés, il est essentiel de bien distinguer ce qui relève du phénomène physique responsable des inondations de ses conséquences en terme de dégâts. A cet effet, le Ministère de l'Ecologie et du Développement Durable (MEDD) a souhaité la mise en place de deux types d'échelles, la première basée sur les dommages [1], la seconde sur l'intensité du phénomène naturel. Cet article donne les principes généraux qui ont servi à établir la seconde échelle, puis sa traduction nationale pour les inondations. Un exemple d'échelle pour le suivi du risque hydrologique sur le bassin supérieur de l'Hérault est présenté, avec une application opérationnelle pour la gestion de crise en période de crue.

1. Cemagref, U.R. Hydrologie-Hydraulique, 3bis, quai Chauveau, 69336 Lyon Cedex 9 - michel.lang@ @emagref.fr

2. Diren LR, 58, avenue Marie de Montpellier, 34965 Montpellier Cedex 2 — roland.claudet@languedoc-roussillon.environnement.gouv.fr

\section{II — MISE AU POINT D'UNE ÉCHELLE SUR LES INONDATIONS}

L'objectif général d'une échelle d'intensité sur les phénomènes naturels est de pouvoir caractériser sur une grille le niveau d'aléa auquel est exposé un territoire donné et de permettre une classification des événements passés selon leur intensité. L'échelle doit être facilement transférable spatialement, de façon à être comprise de la même façon sur l'ensemble du pays. Dans le domaine des inondations, trois types de variable sont à considérer : a) les paramètres physiques à l'origine des dommages; b) la nature et le montant des dommages; c) la rareté de l'inondation. Chacune de ces dimensions ne peut être considérée indépendamment des autres: la connaissance de la vulnérabilité permet de relier les paramètres physiques de l'inondation avec les dommages, la rareté de l'inondation est relative soit au phénomène physique (aléa), soit aux dommages constatés (risque). Dans les paragraphes suivants, nous discutons des mérites respectifs d'une classification basée sur l'intensité du phénomène physique ou des dommages, ou sur la rareté de l'inondation. 


\section{II.1 Paramètres physiques représentatifs de l'aléa inondation}

\section{II.1.1 Hauteur de submersion et vitesse d'écoulement}

Il semble très délicat de pouvoir résumer la variabilité spatiale des hauteurs de submersion ou des vitesses d'écoulement dans une seule classe d'intensité. Le calcul d'une valeur moyenne (transversalement et longitudinalement) sur l'ensemble de la zone inondée n'apporte qu'une information très réductrice sur le caractère dommageable de la crue. La sélection de la valeur maximale de la hauteur de submersion présente peu d'intérêt dans le lit mineur de la rivière. Elle soulève des difficultés méthodologiques dans le lit majeur, car la configuration topographique varie suivant le profil longitudinal de la rivière, et d'une rivière à une autre. La vitesse d'écoulement est un paramètre difficile à estimer. Si des valeurs locales peuvent être reconstituées, le calcul détaillé du champ des vitesses nécessite une modélisation hydraulique complexe, inadaptée à une procédure systématique de caractérisation du niveau d'intensité des crues. L'utilisation de formules hydrauliques simples ne fournit qu'une estimation de la vitesse moyenne dans une section d'écoulement et ne donne pas accès aux vitesses locales d'écoulement. En période de crue, celles-ci peuvent être bien différentes de la vitesse moyenne, lorsque les écoulements sont concentrés sur des portions réduites de la section en travers. Comme pour les hauteurs, le calcul d'une vitesse moyenne ou d'une valeur maximale pour l'ensemble de la crue présente peu de pertinence.

\section{II.1.2 Débit maximal de crue}

A l'inverse des deux paramètres précédents, le débit de la crue est une variable intégratrice, représentative des écoulements collectés sur l'ensemble du bassin versant considéré. L'intensité du débit renseigne sur l'importance de la crue, mais elle ne donne pas directement d'indication sur l'intensité potentielle des dommages en un point donné. On peut toutefois traduire la valeur de ce débit en champ d'inondation (avec une cartographie des hauteurs et des vitesses de submersion), à partir de la topographie de la zone inondée et une modélisation hydraulique des écoulements. Une échelle graduée en $\mathrm{m}^{3} / \mathrm{s}$ serait difficilement transférable d'un site à un autre, car la valeur du débit est fortement dépendante de la superficie du bassin versant et de la réponse aux précipitations de ce bassin. Cette variabilité n'est que partiellement réduite avec l'emploi d'une graduation en débit spécifique $\left(\mathrm{m}^{3} / \mathrm{s} \cdot \mathrm{km}^{2}\right)$ ou pseudo-spécifique $\left(\mathrm{m}^{3} / \mathrm{s} \cdot \mathrm{km}^{1.6}\right)$. A titre d'exemple $^{3}$, le débit de crue des cours d'eau de la région Centre varie généralement de 6 à 40 1/s.ha ${ }^{1.6}$, mais il peut dépasser 100 sur la partie amont des bassins de la Loire et de l'Allier (crues cévenoles). L'emploi d'une telle échelle nécessiterait d'adapter les barreaux en fonction de la typologie des bassins et de décliner plusieurs échelles suivant la gamme de superficie considérée.

\section{II.1.3 Précipitations à l'origine de la crue}

La lame d'eau précipitée sur le bassin versant considéré donne une bonne idée de l'origine météorologique du phénomène d'inondation. Mais cette information n'est pas suffi-

3. Communication personnelle de M. Ghio (ex. Diren Centre) sante pour caractériser l'intensité des inondations, car l'état initial de saturation du bassin versant joue un rôle important. De plus, l'emploi d'une échelle basée sur la pluviométrie nécessiterait de régionaliser les barreaux pour tenir compte de la climatologie du secteur considéré. A titre d'exemple, le dépassement d'un seuil de pluviométrie de $100 \mathrm{~mm}$ en 24 heures devrait correspondre à une forte intensité dans le Nord de la France, alors que ce type d'événement arrive couramment dans le sud.

\section{II.2 Dommages constatés ou potentiels d'une inondation}

L'intensité d'un phénomène naturel peut être mesurée d'après les effets observés ou les dommages potentiels sur des enjeux type. C'est souvent l'approche retenue pour des phénomènes naturels où l'intensité physique ne peut être décrite simplement (volcanisme, mouvements de terrain). Dans le cas d'une échelle d'intensité du phénomène d'inondation, il s'agirait de relier de façon simple le niveau des dommages avec celui de l'aléa pour un certain nombre d'enjeux type. Cette approche est notamment reprise par les historiens [2,3], lorsqu'ils classent les crues anciennes d'après les conséquences répertoriées dans les fonds d'archive. La difficulté de ce type d'échelle est de faire abstraction de l'évolution dans le temps de la vulnérabilité ou des conditions d'écoulement. Des analyses menées sur des séries historiques de crue en France ou en Espagne [4] ont notamment montré que la répartition des crues dans les différentes classes de dommage pouvait être influencée par les aménagements de la rivière au cours du temps, ou par le développement de l'urbanisation de cités proches des cours d'eau.

\section{II.3 Probabilité d'occurrence de l'inondation}

Il est d'usage chez les hydrologues de caractériser l'intensité d'une inondation d'après la période de retour relative au dépassement du débit de la pointe de crue. On peut reprendre ce principe pour classer sur une échelle un événement donné en comparant le débit de la crue avec une série de débits de référence, chacun étant relatif à une période de retour standard. L'avantage est de raisonner directement sur le phénomène physique, à partir d'une variable intégratrice (le débit), tout en s'affranchissant des problèmes d'échelle spatiale et d'effet de site (topographie, vulnérabilité).

Ce type d'échelle présente toutefois deux difficultés. Il ne répond pas directement à la question posée de graduer les crues en fonction de l'intensité physique des paramètres responsables des dommages. On peut cependant associer des dommages prévisibles à chaque classe de débit en fréquence, sachant qu'il existe des relations entre le gabarit du lit mineur et le régime des crues, que les ouvrages hydrauliques sont dimensionnés par rapport à un débit de référence et que les phénomènes d'érosion de berge ou de transport solide apparaissent au-delà d'un certain seuil de débit. De plus, le concept de période de retour n'est pas facile à appréhender pour le grand public. Le débit de la crue centennale par exemple, qui a une chance sur 100 chaque année d'être dépassé, est souvent interprété à tort comme celui d'une crue ne revenant qu'une seule fois par siècle. Les inquiétudes en 
cours sur la crue centennale de 1910 sur la Seine à Paris, susceptible de revenir prochainement, sont à cet égard significatives.

\section{III — PROJET NATIONAL D'ÉCHELLE D'INTENSITÉ SUR LES ALÉAS NATURELS}

Le MEDD a passé en 2001 une commande au bureau d'étude GéoSciences Consultant, associé à Météo-France et au Cemagref, pour la mise au point d'une série d'échelles d'intensité [5] sur les différents aléas naturels météorologiques, gravitaires, volcaniques... L'objectif est de disposer d'une mesure d'intensité des phénomènes naturels, indépendante de leur localisation, qui permette de suivre l'évolution dans le temps de la vulnérabilité, en comparant les endommagements observés pour des événements de même intensité. A l'issue d'un travail bibliographique et d'une enquête auprès d'experts [6], il a été décidé de caractériser les inondations à partir d'une échelle à quatre niveaux basée sur la fréquence de la crue et de décliner localement une échelle en trois niveaux d'intensité physique de submersion. Le niveau de danger prévisible dépend à la fois de la rareté de la crue et de la traduction locale de l'inondation suivant la configuration topographique. Le nombre de classes a été volontairement réduit, pour les fréquences afin de positionner plus facilement un événement dans une des classes, et pour l'intensité de submersion, car trois classes d'intensité (faible, moyenne, fort) apparaissaient comme suffisantes pour discriminer les situations.

\section{III.1 Echelle probabiliste de gravité des inondations}

Le tableau 1a présente la première partie de l'échelle basée sur des critères hydrologiques. Le débit de pointe de la crue (ou son volume, pour des crues lentes) est comparé avec une des trois valeurs de référence : 1) $Q_{0}$ est le débit associé au début des débordements gênants. Il est estimé en fonction de la connaissance locale des inondations, ou à partir du débit associé au dépassement de la cote d'alerte (si le bassin fait l'objet d'une annonce des crues), ou à partir du débit de période de retour 2 ans. 2) $Q(10)$ est le débit décennal de crue. Il est estimé directement à partir de séries d'observations en débit, ou à partir de synthèses régionales sur les crues (cf. Diren). 3) $Q_{\mathrm{PHEC}}$ est le débit associé aux Plus Hautes-Eaux Connues (PHEC). Il est estimé à partir de la connaissance du niveau des PHEC et d'une conversion hauteur-débit, ou à partir d'une étude hydrologique fournissant la valeur du débit de crue centennal. Lorsque les informations en débit sont peu précises ou indisponibles, on peut raisonner sur la cote maximale de crue $H$ ou le ratio $R$ de la superficie inondée par la crue avec la superficie inondée par la crue la plus forte connue. Cette seconde variable n'est pas à utiliser lorsque la superficie inondée varie peu avec le débit (cas des secteurs avec une vallée soit très large, soit très encaissée). Le choix des trois niveaux de référence $Q_{0}$, $Q(10)$ et $Q_{\text {PHEC }}$ a été fait d'après des critères hydrologiques (variation logarithmique des quantiles de débit) et des critères d'usage (critère d'événement décennal pour la déclaration d'état de catastrophe naturelle, crue centennale ou crue historique pour les Plans de Prévention du Risque d'Inondation, PPRI).

Afin de permettre une meilleure compréhension des trois seuils précédents (période de retour 2, 10 et 100 ans) nous avons repris un mode de représentation graphique issu d'un travail de thèse sur l'évaluation du consentement à payer pour bénéficier de protections contre les inondations [7]. L'idée est de faire appréhender le comportement aléatoire des crues, en donnant la probabilité que la crue la plus forte observée sur une période considérée ( 1 an, 20 ans et 50 ans) appartienne à chacune des quatre classes d'intensité. On voit par exemple que la crue centennale (classe 4), qui revient en moyenne une fois par siècle, a une chance sur 100 d'être dépassée chaque année, environ une chance sur cinq en 20 ans et un peu moins d'une chance sur deux en 50 ans, ce qui est loin d'être négligeable à l'échelle de la vie humaine ou de la durée de vie des biens exposés aux inondations. Les figures de la deuxième colonne représentent une urne constituée de boules dont l'effectif en niveaux de gris est proportionnel à la probabilité de chacune des classes d'intensité. On voit aisément que le risque d'observer une crue d'intensité élevée à exceptionnelle augmente au fur et à mesure de l'assombrissement de l'urne.

Le tableau $1 \mathrm{~b}$ présente la seconde partie de l'échelle basée sur les dommages potentiels sur des enjeux type, avec l'avertissement qu'il ne s'agit que de dommages moyens prévisibles, susceptibles de variations spatiales en fonctions de spécificités locales : les dommages réels peuvent être plus élevés (forte vulnérabilité, rupture de digue, longue durée de submersion) ou moins importants (zones d'expansion naturelle, ouvrages de protection...).

La caractérisation finale d'un événement de crue sera faite à l'aide de deux informations, l'extension spatiale des inondations et la valeur de la classe d'intensité associée à cette échelle spatiale. On distinguera quatre classes d'extension $\left(E\right.$ en $\left.\mathrm{km}^{2}\right): 1$. faible : $E<50 ; 2$. moyenne : $50<E<500$; 3. forte : $500<E<5000$; 4. très forte : $E>5000$.

\section{III.2 Echelles d'intensité de submersion}

Ce second type d'échelle est basé sur les paramètres physiques à l'origine des dommages. Cette information, fortement variable selon la position du point considéré, pourra être reportée sur une carte (cf. atlas des zones inondables, carte d'aléa d'un PPRI) pour bien appréhender la variabilité spatiale des phénomènes. Nous proposons deux échelles d'intensité suivant la dynamique de la crue, afin de bien distinguer les effets des crues lentes (tab. 2a), avec un faible gradient de montée et une durée de crue supérieure à la journée, de ceux des crues rapides (tab. 2b). Pour les premières on dispose d'un délai suffisant pour déplacer des biens sensibles, par contre il est assez difficile d'empêcher l'eau de pénétrer dans les habitations. Les dommages sont en général plus élevés pour les biens immobiliers avec les crues lentes que pour les crues rapides, et inversement pour les biens mobiliers. Le cas particulier d'une submersion brutale liée à une rupture de digue ou d'embâcle doit être traité comme celui d'une crue rapide, dans la zone concernée par ce phénomène. 
Tableau 1a: Echelle probabiliste de gravité des inondations : critères hydrologiques

\begin{tabular}{|c|c|c|c|c|c|c|}
\hline \multicolumn{3}{|c|}{ Classe d'intensité } & 1. Faible & 2. Moyenne & 3.Elevée & 4. Exceptionnelle \\
\hline \multicolumn{3}{|c|}{ Niveau de gris } & $10 \%$ & $30 \%$ & $60 \%$ & $100 \%$ \\
\hline \multirow{3}{*}{\multicolumn{2}{|c|}{$\begin{array}{c}\text { Paramètres } \\
\text { hydrologiques }\end{array}$}} & Débit $Q$ & $Q<Q_{0}$ & {$\left[Q_{0} ; Q(10)\right]$} & {$\left[Q(10) ; Q_{P H E C}\right]$} & $Q>Q_{P H E C}$ \\
\hline & & Hauteur $H$ & $H<H_{0}$ & {$\left[H_{0} ; H(10)\right]$} & {$[H(10) ; P H E C]$} & $H>P H E C$ \\
\hline & & $\begin{array}{c}\text { Ratio } \boldsymbol{R} \\
\text { (superficie inondée) }\end{array}$ & $<0,1$ & {$[0,1 ; 0,5]$} & {$[0,5 ; 1]$} & $>1$ \\
\hline \multirow{3}{*}{$\begin{array}{l}\text { Probabilité que la } \\
\text { plus forte crue } \\
\text { sur } N \text { années } \\
\text { appartienne à la } \\
\text { classe d'intensité } \\
1,2,3 \text { ou } 4\end{array}$} & $\begin{array}{l}N= \\
1 \text { an }\end{array}$ & & 0,50 & 0,40 & 0,09 & 0,01 \\
\hline & $\begin{array}{c}N= \\
20 \text { ans }\end{array}$ & & $\approx 0$ & 0,12 & 0,70 & 0,18 \\
\hline & $\begin{array}{c}N= \\
50 \text { ans }\end{array}$ & & $\approx 0$ & 0,01 & 0,60 & 0,39 \\
\hline
\end{tabular}

Tableau 1b: Echelle probabiliste de gravité des inondations : dommages prévisibles

\begin{tabular}{|c|c|c|c|c|c|}
\hline \multicolumn{2}{|c|}{ Classe d'intensité } & 1. Faible & 2. Moyenne & 3. Elevée & 4. Exceptionnelle \\
\hline \multirow{2}{*}{$\begin{array}{l}\text { Effets pré- } \\
\text { visibles sur } \\
\text { les enjeux }\end{array}$} & Personnes & I & $\begin{array}{c}\text { Risque très faible, sauf par } \\
\text { imprudence ou proximité } \\
\text { immédiate de la rivière }\end{array}$ & $\begin{array}{l}\text { Risque de noyade pour des } \\
\text { personnes surprises par la } \\
\text { crue (à l'intérieur de l'habi- } \\
\text { tation ou d'une voiture) }\end{array}$ & $\begin{array}{l}\text { Pertes humaines proba- } \\
\text { bles, surtout en cas } \\
\text { de crue rapide }\end{array}$ \\
\hline & Bâtiments & I & $\begin{array}{l}\text { Inondation de bâtiments } \\
\text { près du lit mineur; de } \\
\text { caves en milieu urbain ; de } \\
\text { campings }\end{array}$ & $\begin{array}{l}\text { Dommages mobiliers impor- } \\
\text { tants pour les bâtiments } \\
\text { exposés ; endommagement } \\
\text { possible de bâtiments. }\end{array}$ & $\begin{array}{l}\text { Dommages mobiliers } \\
\text { et immobiliers élevés }\end{array}$ \\
\hline \multirow{3}{*}{$\begin{array}{l}\text { Facteurs } \\
\text { aggravants : } \\
\text { * forte } \\
\text { extension } \\
\text { spatiale } \\
\text { * crue } \\
\text { rapide } \\
\text { * forte } \\
\text { durée de } \\
\text { submersion }\end{array}$} & $\begin{array}{c}\text { Infra- } \\
\text { structures }\end{array}$ & $\begin{array}{l}\text { Submer- } \\
\text { sions } \\
\text { minimes }\end{array}$ & $\begin{array}{c}\text { Coupures de routes secon- } \\
\text { daires ou voies sur berge ; } \\
\text { Mise en alerte de } \\
\text { l'annonce des crues et } \\
\text { mobilisation des services } \\
\text { chargés des secours et des } \\
\text { réseaux }\end{array}$ & $\begin{array}{l}\text { Désordres significatifs sur } \\
\text { le réseau routier secondaire } \\
\text { et quelques routes } \\
\text { nationales ; désordres sur les } \\
\text { autres réseaux (eau, électri- } \\
\text { cité, gaz, chauffage, } \\
\text { assainissement ...) }\end{array}$ & $\begin{array}{l}\text { Désordres importants } \\
\text { sur le réseau routier, } \\
\text { voies ferrées et autres } \\
\text { réseaux ; situation } \\
\text { de crise dès que l'exten- } \\
\text { sion spatiale est } \\
\text { importante }\end{array}$ \\
\hline & $\begin{array}{c}\text { Espaces } \\
\text { naturels } \\
\text { et agricoles }\end{array}$ & $\begin{array}{l}\text { Débor- } \\
\text { dements } \\
\text { limités } \\
\text { aux zones } \\
\text { humides }\end{array}$ & $\begin{array}{l}\text { A proximité du lit mineur, } \\
\text { inondation de zones culti- } \\
\text { vées et perte éventuelle de } \\
\text { bétail dans les pâturages }\end{array}$ & $\begin{array}{c}\text { Dommages conséquents } \\
\text { (terres cultivées, vignes, } \\
\text { vergers ...) }\end{array}$ & $\begin{array}{l}\text { Dommages importants } \\
\text { sur les cultures et certai- } \\
\text { nes exploitations } \\
\text { agricoles }\end{array}$ \\
\hline & $\begin{array}{l}\text { Rivières } \\
\text { et ouvrages } \\
\text { fluviaux }\end{array}$ & $\begin{array}{c}\text { Débor- } \\
\text { dements } \\
\text { sans effet } \\
\text { notable }\end{array}$ & $\begin{array}{l}\text { Désordres ponctuels sur la } \\
\text { rivière (berges, digues) }\end{array}$ & $\begin{array}{l}\text { Désordres sur des linéaires } \\
\text { significatifs de la rivière } \\
\text { (jusqu'à } 100 \mathrm{~m} \text { ) ; piles } \\
\text { de ponts attaquées ou } \\
\text { sur creusées, indices } \\
\text { de transport solide }\end{array}$ & $\begin{array}{l}\text { Transformation impor- } \\
\text { tante de la morphologie } \\
\text { fluviale ; destruction } \\
\text { d'infrastructures sur } \\
\text { plusieurs centaines } \\
\text { de } \mathrm{m} \text {; ponts emportés }\end{array}$ \\
\hline
\end{tabular}


Tableau 2a: Echelle d'intensité de submersion (crues lentes, durée de crue supérieure à 1 jour)

\begin{tabular}{|c|c|c|c|c|}
\hline \multicolumn{2}{|c|}{ Classe d'intensité } & 1. Faible & 2. Moyenne & 3. Forte \\
\hline \multicolumn{2}{|c|}{ Niveau de gris } & $10 \%$ & $50 \%$ & $100 \%$ \\
\hline \multirow{2}{*}{$\begin{array}{l}\text { Paramètres } \\
\text { Physiques }\end{array}$} & Hauteur $H(\mathrm{~m})$ & $H<0,5$ & $0,5<H<1$ à 2 & $\mathrm{H}>1$ à 2 \\
\hline & Durée $d$ & Environ 1 jour & Quelques jours & Plusieurs semaines \\
\hline \multirow{2}{*}{$\begin{array}{l}\text { Dommages } \\
\text { potentiels }\end{array}$} & Personnes & $\begin{array}{c}\text { Mobilité entravée, } \\
\text { aléatoire si vitesse } \\
\text { de courant } \\
\text { moyenne }\end{array}$ & Mobilité aléatoire & Danger de noyade \\
\hline & Bâtiments & $\begin{array}{l}\text { Eau dans bâtiment } \\
\text { peut être maîtrisée. } \\
\text { Sous-sol endom- } \\
\text { magé }\end{array}$ & $\begin{array}{l}\text { Eau dans bâtiment ; Fenêtres } \\
\text { peuvent se briser ; retraite } \\
\text { vers étage supérieur possible ; } \\
\text { Sous-sol fortement endom- } \\
\text { magé et RdC atteint }\end{array}$ & $\begin{array}{l}\text { RdC et caves entièrement } \\
\text { inondés ; chemin de fuite ou } \\
\text { vers le toit coupé. Destruction } \\
\text { possible de bâtiments anciens } \\
\text { en brique, pisée et/ou pierre }\end{array}$ \\
\hline \multirow{3}{*}{$\begin{array}{l}\text { Facteurs } \\
\text { aggravants : } \\
* \text { forte extension } \\
\text { spatiale }\end{array}$} & Infra-structures & Route coupée & $\begin{array}{c}\text { Chaussée endommagée } \\
\text { si } V>1 \mathrm{~m} / \mathrm{s}\end{array}$ & $\begin{array}{l}\text { Chaussée fortement endom- } \\
\text { magée si } V>1 \mathrm{~m} / \mathrm{s}\end{array}$ \\
\hline & $\begin{array}{c}\text { Espaces } \\
\text { naturels } \\
\text { et agricoles }\end{array}$ & $\begin{array}{l}\text { Faible impact, sauf } \\
\text { maraîchage, vignes }\end{array}$ & $\begin{array}{l}\text { A proximité du lit mineur, } \\
\text { dommages sur des zones cul- } \\
\text { tivées et perte éventuelle de } \\
\text { bétail dans les pâturages }\end{array}$ & $\begin{array}{c}\text { Dommages conséquents } \\
\text { (terres cultivées, vignes, } \\
\text { vergers ....). }\end{array}$ \\
\hline & $\begin{array}{l}\text { Rivières } \\
\text { et ouvrages } \\
\text { fluviaux }\end{array}$ & Impact limité & $\begin{array}{l}\text { Désordres possibles sur les } \\
\text { ponts si vitesse de courant et } \\
\text { obstruction de matériaux. } \\
\text { Désordres locaux dans les } \\
\text { digues ou berges. }\end{array}$ & $\begin{array}{c}\text { Ponts submergés ou emportés } \\
\text { si vitesse de courant ; digues } \\
\text { détruites }\end{array}$ \\
\hline
\end{tabular}

Tableau 2b: Echelle d'intensité de submersion (crues rapides, durée de crue inférieure à 1 jour)

\begin{tabular}{|c|c|c|c|c|}
\hline \multicolumn{2}{|c|}{ Classe d'intensité } & 1. Faible & 2. Moyenne & 3. Forte \\
\hline \multicolumn{2}{|c|}{ Niveau de gris } & $10 \%$ & $50 \%$ & $100 \%$ \\
\hline \multirow{3}{*}{$\begin{array}{l}\text { Paramètres } \\
\text { Physiques }\end{array}$} & $\begin{array}{l}\text { Gradient } G \text { de } \\
\text { montée des } \\
\text { eaux }\end{array}$ & $G<1 \mathrm{~m} / 12 \mathrm{~h}$ & $1 \mathrm{~m} / 12 \mathrm{~h}<G<1 \mathrm{~m} / 1 \mathrm{~h}$ & $G>1 \mathrm{~m} / 1 \mathrm{~h}$ \\
\hline & $\begin{array}{l}\text { Hauteur } H \\
\text { Vitesse } V\end{array}$ & $\begin{array}{c}H \text { ou } V . H<0,5 \\
H(\mathrm{~m}) \text { et } V . H\left(\mathrm{~m}^{2} / \mathrm{s}\right)\end{array}$ & $0,5<H$ ou V.H $<1$ à 2 & $H$ ou $V . H>1$ à 2 \\
\hline & $\begin{array}{l}\text { Profondeur de } \\
\text { brèche } p\end{array}$ & $\begin{array}{c}p<0,5 \mathrm{~m} \\
\text { (rupture de digue) }\end{array}$ & $0,5<p<2$ & $p>2$ \\
\hline \multirow[b]{2}{*}{$\begin{array}{l}\text { Dommages } \\
\text { potentiels }\end{array}$} & Personnes & Danger mineur & Danger d'emportement & Danger de noyade \\
\hline & Bâtiments & Dégâts mineurs & $\begin{array}{l}\text { Destruction de maisons peu } \\
\text { résistantes (bois, préfabriqué, } \\
\text { sans fondation) ; dommages à } \\
\text { des bâtiments en béton armé }\end{array}$ & $\begin{array}{l}\text { Destruction de bâtiments en } \\
\text { béton armé (mur, fondations) }\end{array}$ \\
\hline \multirow{3}{*}{$\begin{array}{l}\text { Facteurs } \\
\text { aggravants : } \\
\text { * forte extension } \\
\text { spatiale } \\
\text { * effet de surprise } \\
\text { (crue la nuit, } \\
\text { absence d'alerte) }\end{array}$} & Infra-structures & Route coupée & $\begin{array}{c}\text { Chaussée endommagée ; } \\
\text { véhicule léger emporté ; } \\
\text { réseau enterré ponctuellement } \\
\text { détruit }\end{array}$ & $\begin{array}{c}\text { Chaussée emportée ; véhicule } \\
\text { lourd emporté ; réseau enterré } \\
\text { détruit ; aérien en partie } \\
\text { détruit }\end{array}$ \\
\hline & $\begin{array}{l}\text { Espaces natu- } \\
\text { rels et agricoles }\end{array}$ & $\begin{array}{l}\text { Faible impact, sauf } \\
\text { maraîchage vignes }\end{array}$ & $\begin{array}{c}\text { Dommages de zones cultivées } \\
\text { et perte éventuelle de bétail } \\
\text { dans les pâturages }\end{array}$ & $\begin{array}{c}\text { Dommages conséquents } \\
\text { (terres cultivées, vignes, } \\
\text { vergers ...) }\end{array}$ \\
\hline & $\begin{array}{l}\text { Rivières } \\
\text { et ouvrages } \\
\text { fluviaux }\end{array}$ & Impact limité & Erosion de berges & $\begin{array}{l}\text { Tronçons significatifs avec } \\
\text { forte érosion des berges ; } \\
\text { désordres sur les culées } \\
\text { et ponts ; divagation possible } \\
\text { du lit. }\end{array}$ \\
\hline
\end{tabular}




\section{IV — ÉCHELLE DE RISQUE HYDROLOGIQUE SUR L'HÉRAULT AMONT}

\section{IV.1 Conception générale du système d'information}

\section{IV.1.1 Présentation}

Le système de gestion de crise construit sur l'Hérault amont, pour 9 communes du secteur de Ganges ${ }^{4}$, a un élément-clé : l'échelle de gravité du risque hydrologique.

Sous la dénomination "plans d'interventions graduées », ce système comprend une partie cartographique opérationnelle (zones inondées pour chaque niveau de l'échelle de gravité) plus des fiches-réflexes. Destiné à être intégré dans le plan communal de sauvegarde, il répond à trois objectifs majeurs :

- traduire dans un document de référence les connaissances et les savoir-faire accumulés par tous les acteurs de la gestion de crise ;

- donner aux services opérationnels un outil d'aide à la décision, notamment pour informer de façon anticipée les populations exposées aux risques et lancer les actions nécessaires sur le terrain ;

- différencier les interventions par secteurs hydrographiques et par communes selon la gravité attendue ou constatée des événements de crue et d'inondation et en tenant compte des différences de vulnérabilité des biens et des personnes.

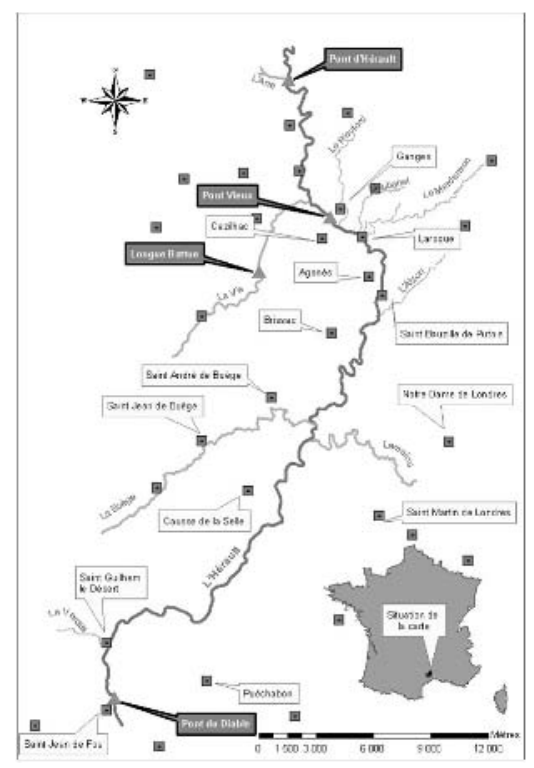

Figure 1 : Secteur amont de l'Hérault.

\section{IV.1.2 Une architecture commune aux acteurs «amont-aval» de la gestion de crise}

Cette architecture (fig. 2) trouve sa logique dans une double nécessité : agir par anticipation et à bon escient, mais en utilisant entre l'ensemble des acteurs un langage et des mes-

4. Agonès, Brissac, Cazilhac, Causse de la Selle, Ganges, St-Bauzille de Putois, Laroque, St-Guilhem le Désert, St Martin de Londres. sages suffisamment codifiés pour qu'il n'y ait aucune ambiguïté sur leurs contenus et leurs portées.

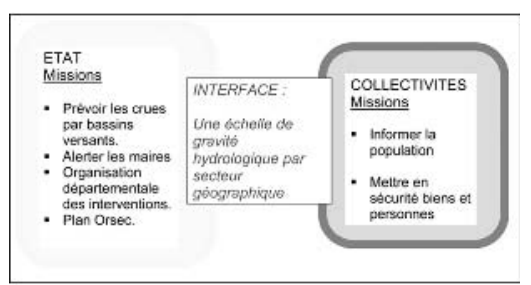

Figure 2 : L'échelle de gravité au cœur du système d'information.

Cela suppose bien sûr de disposer d'un système de prévision météorologique et hydrologique efficace. Mais si ce n'est pas encore le cas, rien n'interdit de mettre en place cette architecture en prenant appui sur une pratique d'appréciation locale et en temps réel des précipitations et des niveaux d'eau. Ce sera notamment le cas pour les bassins versants de petite taille, encore peu instrumentés, où toute prévision est encore difficile à établir.

\section{IV.2 L'étude des plans d'interventions graduées}

\section{IV.2.1 Les principes}

L'objectif étant de dresser des scénarios pré-établis intégrables dans un plan, l'étude consiste à faire émerger le système de correspondances aléa-risque-gestion de crise, tel qu'il est vécu mais aussi tel qu'il pourrait devenir avec une plus grande anticipation et l'instauration d'un certain nombre de niveaux de gravité.

D'où l'idée d'utiliser une échelle de gravité des crues pour un secteur géographique donné, apte à être traduite localement (compte tenu de la vulnérabilité de chaque commune) en cartes d'inondations et plan de gestion de crise, et apte à servir de mode commun de langage avant et pendant la crise.

Autour de cette échelle naît le plan d'interventions graduées, qui est de fait un plan de secours inondations (fig. 3). Une enquête est nécessaire, qui peut prendre appui sur la trame de questionnaire proposée dans le Guide méthodologique «plan local de gestion de crise » édité par la Diren Midi-Pyrénées, qui comprend par territoire communal un questionnaire à l'attention de l'ensemble des acteurs de gestion de crise, en 5 points : état du système d'alerte, état du système de secours, analyse et gestion de la crise, recensement de l'information existante et état de la connaissance du risque.

\section{IV.2.2 Déroulement de l'étude}

Une étude de faisabilité a été confiée au printemps 2001 par la DIREN Languedoc-Roussillon à l'ENGREF, centre de Montpellier, en partenariat avec le service d'annonce des crues de l'Hérault. La commande passée à un groupe d'étudiants de $1^{\text {re }}$ année était de tester les conditions de mise en œuvre de plans de secours inondations pour les communes du secteur de Ganges, en mettant à profit le modèle indicateur de risques développé sur ce cours d'eau calé sur trois niveaux de gravité des crues à la station de référence de Ganges. 


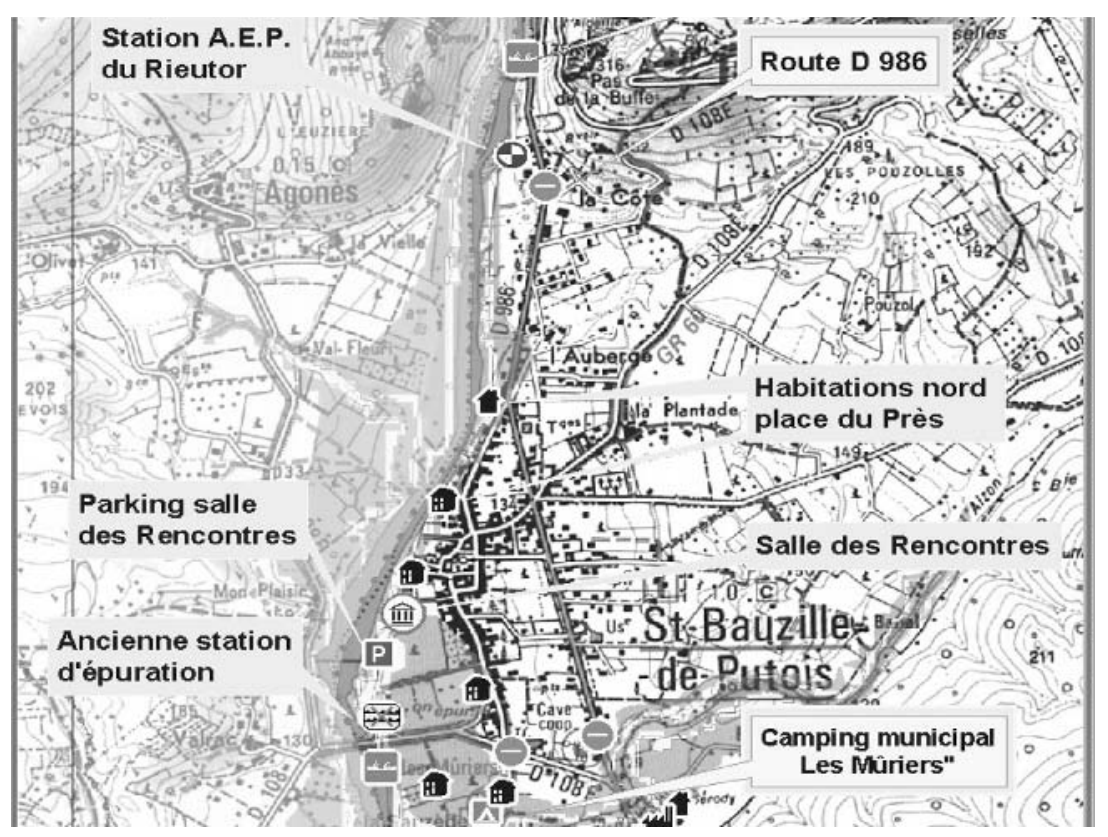

Figure 3 : Plan d'intervention à St-Bauzille-de-Putois.

Les résultats de cette pré-étude ayant été tout à fait concluants, il a été décidé dès l'automne 2001 de passer à la réalisation définitive des plans de secours. Ont été choisis pour cette étude à caractère méthodologique sous maîtrise d'ouvrage DIREN : l'Ecole des Mines de St-Etienne ${ }^{5}$ (Centre site - Sciences, information et technologies pour l'environnement), associée pour la partie initiale d'enquête à un cabinet d'ergonomie, Masseron et Trentin, de Toulouse et pour leur savoir-faire en matière de plan de secours incendies de forêt le CEREN (Centre d'études du risque de l'entente méditerranéenne de Valabre-Gardanne).

Un comité de pilotage a été constitué avec les apports de la direction départementale de l'équipement de l'Hérault (centre d'annonce des crues et subdivision de Ganges), du centre des pompiers de Ganges, de la brigade de gendarmerie de Ganges, du Conseil Général (services de l'hydraulique et des routes) et du SIVOM de Ganges-Le Vigan.

\section{IV.2.3 L'échelle de gravité du risque hydrologique}

Pour le bassin versant de l'Hérault amont, secteur de Ganges, une échelle de gravité a été créée de fait en 2000 à l'occasion de la construction de modèles de prévision de crues, nommés «indicateur de risques ${ }^{6}$. L'objectif était de disposer d'outils de prévision robustes capables d'une part d'utiliser en temps réel les lames d'eau de précipitations issues des images radar et des stations au sol et d'autre part de permettre d'anticiper sur les risques de crues pour 3 niveaux de gravité différents. Choisis à dire d'experts compte tenu de la vulnérabilité des communes proches des stations de référence, ces niveaux se sont trouvés calés en moyenne sur les fréquences de crues

5. Etude EMSE pilotée par Roland Dechomets, avec l'appui de Mireille Batton-Hubert.

6. Au nombre de dix pour des bassins à crues rapides du Languedoc-Roussillon, ces indicateurs ont tous été élaborés sur trois niveaux de risques. Sous maîtrise d'ouvrage Diren LR, les études de faisabilité générale puis de construction des modèles ont été confiées à la société Rhéa, réalisation par Jean-Luc Nuel.

\begin{tabular}{|c|c|c|}
\hline \multicolumn{3}{|c|}{$\begin{array}{l}\text { Crues historiques } \\
\text { débits - niveaux - temps de retour }\end{array}$} \\
\hline 1 à 2 ans & $350 \mathrm{~m} 3 / \mathrm{s}$ & $2,8 \mathrm{~m}$ \\
\hline \multirow{3}{*}{ JAUNE } & déc 2000 & $2.8 \mathrm{~m}$ \\
\hline & sept 2002 & $3,9 \mathrm{~m}$ \\
\hline & oct 1999 & $4,2 \mathrm{~m}$ \\
\hline 3 à 5 ans & $750 \mathrm{m3} / \mathrm{s}$ & $4,5 \mathrm{~m}$ \\
\hline \multirow{3}{*}{ ORANGE } & nov 1997 & $4,7 \mathrm{~m}$ \\
\hline & oct 1995 & $5,0 \mathrm{~m} ; 5,4 \mathrm{~m}$ \\
\hline & sept 1995 & $5,7 \mathrm{~m}$ \\
\hline 10 à 20 ans & $1100 \mathrm{~m} 3 / \mathrm{s}$ & $6,0 \mathrm{~m}$ \\
\hline \multirow{3}{*}{ ROUGE } & déc 1997 & $6,8 \mathrm{~m}$ \\
\hline & nov 1994 & $7.9 \mathrm{~m}$ \\
\hline & oct 1958 & $8,0 \mathrm{~m}$ \\
\hline
\end{tabular}

Figure 4 : Crues historiques de référence.

suivantes : 1) annuelle à bi-annuelle, 2) triennale à quinquennale, 3) décennale ou plus.

La confirmation de cette échelle de gravité, comme clé de voûte des plans d'interventions, a été discutée avec l'ensemble des services opérationnels locaux. Cette validation s'est faite selon trois critères principaux : l'horizon d'anticipation, un nombre raisonnable et une différenciation suffisante de scénarios d'interventions, enfin la compréhension aisée des futurs messages entre l'ensemble des utilisateurs.

Le comité de pilotage a validé le choix d'une échelle à 3 niveaux de gravité en recommandant de les codifier à l'aide des mêmes couleurs que celles de l'échelle de vigilance météorologique. Cette échelle de risques trouve son illustration par les niveaux d'eau atteints par les crues à la station de Ganges, qui est par ailleurs une station réglementaire pour l'annonce des crues. 


\section{IV.2.3 Les fiches réflexes}

Ce plan sera accompagné d'une note succincte d'aide à l'utilisation. Par ailleurs les services d'interventions (pompiers, routes, gendarmerie) auront des documents analogues.
Pour un type de crue donné, les actions à lancer sont priorisées par un indice de vulnérabilité qui classe de fait les interventions de 4 , là où les enjeux humains sont élevés, à 1 , là où seuls les biens matériels sont en cause (fig. 5).

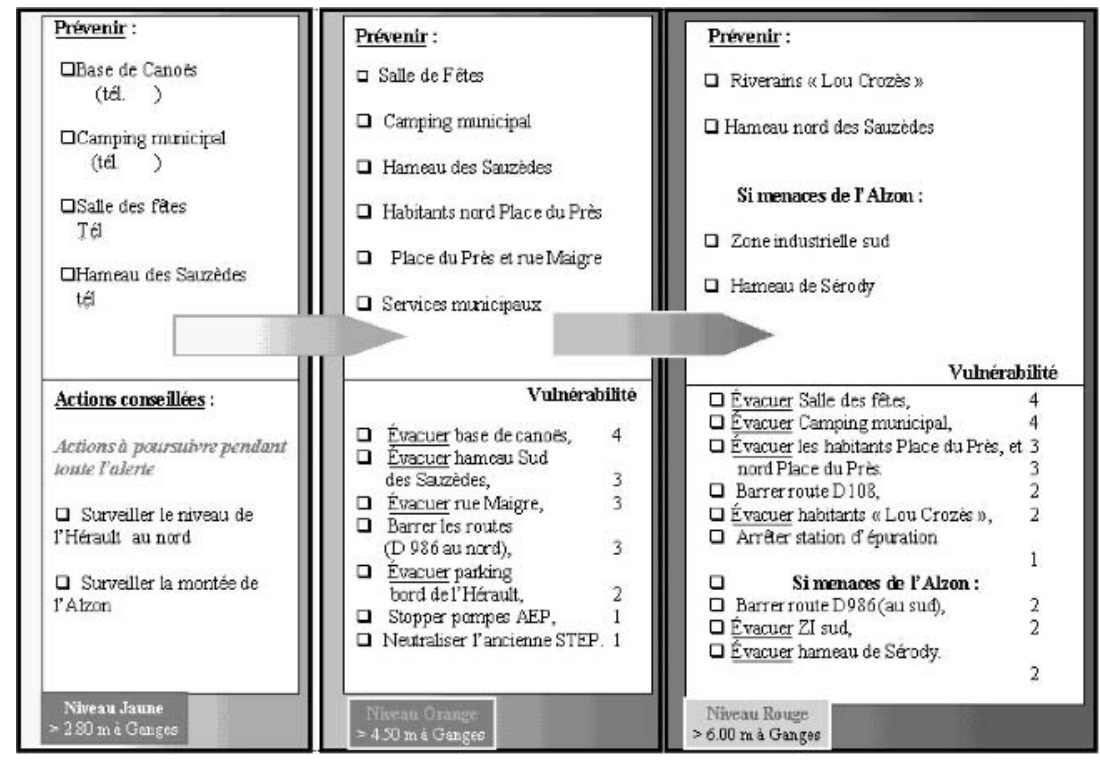

Figure 5 : Fiche réflexe d'intervention.

\section{CONCLUSION}

La gestion du risque d'inondation, que ce soit par des actions de prévention ou de gestion de crise, repose sur une bonne appréhension du phénomène naturel. Il est nécessaire de pouvoir mettre en perspective tout événement de crue à venir ou potentiel par rapport aux autres inondations possibles. La constitution d'une échelle de gravité doit à notre sens respecter les points suivants: 1) utiliser un nombre limité de classes de crues, en analogie avec les autres risques naturels ; 2) adopter une classification générique des événements suivant différents débits de référence, associés chacun à une période retour standard (objectif de transférabilité) ; 3 ) relier chacune des classes de crues à un dommage potentiel pour différents enjeux types; 4) fournir des éléments de compréhension des périodes de retour et des débits de référence, à partir des probabilités et d'événements historiques connus ; 5) décliner spatialement une caractérisation des événements d'après une grille basée sur les paramètres physiques de l'inondation.

\section{BIBLIOGRAPHIE}

[1] Mise (1999). — Echelle de gravité des dommages. Mission d'Inspection Spécialisée Environnement, mai, 1 p.
[2] Ceeur D. (2003). - La maîtrise des inondations dans la plaine de Grenoble (XVII ${ }^{\mathrm{e}}-\mathrm{XX}^{\mathrm{e}}$ siècle) : enjeux techniques, politiques et urbains. Thèse de doctorat, Univ. Pierre Mendès-France, Grenoble, 345 p. +2 volumes d'annexes.

[3] Glaser R., Stangl H., Lang M. (2004). - Floods in Central Europe since $A D 1300$ and their regional context. $175^{\mathrm{e}}$ session du Comité Scientifique et technique «Etiages et crues extrêmes régionaux en Europe. Perspectives historiques ». Congrès de la SHF, Lyon, 28-29 janvier, 63-72.

[4] Barriendos M., Ceeur D., Lang M., Llasat M.C., Naulet R., LEMAITRE F., BARRERA T. (2003). - Stationarity analysis of historical flood series in France and Spain (14th-20th centuries). Natural Hazard and Earth System Science, 3 (6), 583-592.

[5] GéoSciences Consultant, Cemagref, Météo-France (2003). Détermination d'une échelle d'intensité en 5 niveaux par type de phénomènes naturels. Rapport d'étude pour le compte du Ministère de l'Ecologie et du Développement durable, DPPR/ SDPRM, phases 1 à 6 .

[6] Lang M. (2001). - Détermination d'une échelle d'intensité en 5 niveaux par type de phénomènes naturels : Volet Inondations. Etat de l'art et enquête auprès d'experts. Marché MATE $n^{\circ} 01100430013775$ 01, Cemagref Lyon, 39 p.

[7] GRelot F. (2004). - Gestion collective des inondations. Peut-on tenir compte de l'avis de la population dans la phase d'évaluation économique a priori ? Thèse de doctorat, Ensam Paris, Cemagref Lyon, 6 déc., 384 p. 